\title{
Application of Fuzzy Entropy for the Rating of Kinematic Chains
}

\author{
Vallem Srinivasa Rao ${ }^{\# 1}$, Kuchibhotla Mallikarjuna Rao ${ }^{* 2}$, Annambhotla Balaji Srinivasa Rao ${ }^{* 3}$ \\ \# Associate Professor in Mechanical Engineering, Aditya Engineering College \\ Surampalem, E.G.Dt., Andhra Pradesh, India \\ ${ }^{1}$ vallemsrinivasarao@gmail.com \\ "Professor in Mechanical Engineering, College of Engineering, JNTUK \\ Kakinada, E.G.Dt., Andhra Pradesh, India \\ ${ }^{2}$ rangaraokuchibhotla@gmail.com \\ *Professor in Mechanical Engineering, Sri Vasavi Institute of Engg.\&Tech., \\ Nandamuru, Pedana Mandal, Krishna Dist., Andhra Pradesh, India \\ 33.
}

\begin{abstract}
Structural synthesis of kinematic chains is an interesting area for researchers since many years. Selection of best kinematic chain with desired linkage and degree of freedom for a specific industrial purpose i.e. automotive transmission system, robotic manipulators, lifting devices needs systematic synthesis. Many researchers developed algorithms involving lot of computations. Planar kinematic chains can be modeled as fuzzy systems, so that fuzzy logic will be applied. It is necessary to develop quantitative methods to compare the kinematic chains at the conceptual stage of design for characteristics like static and dynamic behaviour, workspace, rigidity etc. These properties have greater meaning when multi degree of freedom chains are considered for application as platform type robots. It is shown that fuzzy entropy can be utilized to compare many kinematic chains with similar linkage characteristics and degree of freedom. In the present work, concept of fuzzy entropy is applied on 8-link 1-dof kinematic distinct chains (16 No.) for the structural comparison and rating. The results are presented in Appendix I.
\end{abstract}

\section{Keywords- Kinematic chain, Uncertainty, Fuzzy entropy, Adjacency matrix, Rating}

\section{INTRODUCTION}

In the structural synthesis of kinematic chains, enumeration and rating are two important areas to be studied. Topology of kinematic chains is useful in comparing them from the structural-error point of view.

AC Rao and CN Rao proposed a method to rate the kinematic chains from the structural error point of view and rated 40 nine-link, two degree of freedom chains using Transmission matrix concept [1, 2]. AC Rao \& CN Rao used the concept of entropy correlated to kinematic chains to select best combination of fixed frame, input and output links [3]. AC Rao proposed a method to rate the kinematic chains based on the structural behaviour which has analogy with Information theory developed in the area of communications [4]. A Novel Method to generate distinct kinematic chains obviating the tedious computations and comparisons to identify isomorphism is developed by ABS Rao et al [5]. GS Bedi, S Sanyal proposed a method based on identifying the chains structural property by using its joint loop connectivity table. They also proposed method for identification of topological characteristics of planar kinematic chains utilizing the connectivity of joints at different levels to detect isomorphism and inversions among 8, 9 and 10-link planar kinematic chains [6, 7]. Ding et al. synthesized whole family of 1-DOF and 3-DOF mechanisms. A general approach for the generation of fractionated topological graphs and the corresponding atlas database for 2- and 3-DOF fractionated planar kinematic chains is proposed [8-10]. Z Chang developed a new method based on Eigen values and Eigen vectors to detect isomorphism in kinematic chains [11]. Hong-Sen Yan, Yu-Ting Chiu presented a complete review on the number synthesis of kinematic chains of mechanisms [12]. TS Mruthyunjaya et al. evolved computer based methods for the structural synthesis and isomorphism detection [13-15]. H Ding and $\mathrm{H}$ Zhen developed loop based methods for detection of isomorphism and rigid sub-chains in the synthesis of kinematic chains [16]. Robert Simoni and Daniel Martins presented selection criteria of kinematic chains based on the concepts of connectivity, redundancy and variety [17]. 
In this paper, a methodology has been developed by using fuzzy entropy as quantitative parameter and illustrated by taking examples for predicting performance of a given kinematic chain without actually carrying out the dimensional synthesis.

\section{FUZZY THEORY}

Fundamental concepts of fuzzy theory [18] applied in this work are introduced. In Boolean algebra, only 2 states are present for any variable. But in many applications, there is a possibility of multiplicity of state variations which need to be represented between 0 and 1. It is very easy to portray the same by using Fuzzy logic. The state variation is also termed as uncertainty, which is denoted with a fraction value between 0 and 1 . It is a measure of membership in a set. Fuzzy index, even though resembling probability to minor extent, it is purely depend on extent of uncertainty of the variable. A fuzziness of 1 denotes certainty that an element belongs to a particular set.

\section{(i) Fuzzy Vector}

Consider the class of students. It is not possible for any student to be always in best position in all aspects i.e. performance in examinations in all subjects, attendance, sports, co-curricular activities, extra-curricular activities. Hence, the performance of a student can be considered as fuzzy parameter. The least performance is 0 $(0 \%)$ and best performance is $1(100 \%)$. For example, performance of three (3) students is $30 \%, 70 \%$ and $95 \%$. These performances can be assigned a fuzzy value $0.30,0.70$ and 0.95 . The parameters to which numbers are assigned in Fuzzy logic are called as 'fits', just like 'bits' in binary logic.

A fuzzy vector in a list will be as: $\mathrm{A}=\left[\begin{array}{lllll}0 & 0.3 & 0.7 & 0.8 & 0.95\end{array}\right]$.

\section{(ii) Crisp Vector}

In the earlier example, a normal candidate weak in all aspects is given ' 0 ' in the class of students and a dynamic candidate with good performance in all aspects is given ' 1 '. In both the cases, the fuzzy parameters or variables become crisp. So, a Crisp vector ' $C$ ' have values '0' and ' 1 ' only. Ex: $C=\left[\begin{array}{lllll}1 & 0 & 0 & 1 & 0\end{array}\right]$.

Two more vectors namely, Nearest crisp vector $\left(C_{n}\right)$ and Farthest crisp vector $\left(C_{f}\right)$ are defined. In both the vectors fits contain ' 0 ' and ' 1 ' only.

For Nearest crisp vector $C_{n}$, fit value will be ' 0 ', if the value of fit ' $f$ ' in primary crisp vector $C$ is $0 \leq f \leq 0.5$ and ' 1 ' if $f>0.5$.

For Farthest crisp vector $\mathrm{C}_{f}$, fit value will be ' 1 ', if the value of fit ' $f$ ' in primary crisp vector $C$ is $0 \leq f \leq 0.5$ and ' 0 ' if $f>0.5$.

For example for the fuzzy vector $\mathrm{A}=\left[\begin{array}{lllll}0 & 0.3 & 0.7 & 0.8 & 0.95\end{array}\right]$,

the nearest crisp vector $C_{n}$ will be $C_{n}=\left[\begin{array}{lllll}0 & 0 & 1 & 1 & 1\end{array}\right]$ and the farthest crisp vector $C_{f}=\left[\begin{array}{llllll}1 & 1 & 0 & 0 & 0\end{array}\right]$.

\section{(iii) Distance between Fuzzy vector \& Crisp vector}

The summation of all the numerical differences to make the given vector A to be equal to crisp vector. In the last example,

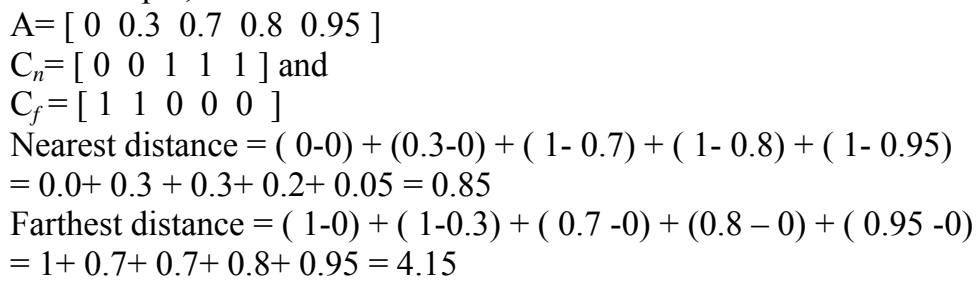

\section{(iv) Pathological Vector}

It is the vector which has all the fit values as 0.5 . It represents the fuzziest (or least certain) possible vector in its n-dimensional space. 


\section{(v) Fuzzy Entropy}

Fuzzy entropy (F) is a measure of uncertainty and is defined for any fuzzy vector A as the ratio of its distance from the nearest crisp vector to that from the farthest vector.

For example, for the fuzzy vector $A=\left[\begin{array}{lllll}0 & 0.3 & 0.7 & 0.8 & 0.95\end{array}\right]$, the distance from the nearest crisp vector is 0.85 while it is 4.15 from the farthest crisp vector. Hence fuzzy entropy $F=0.2048$.

\section{Assigning Fuzzy Parameters for Kinematic chains}

A Kinematic chain is structured with a specified number of links and degree of freedom following the Grubbler's Criterion or Frudenstein equation ${ }^{[10]}$. Each kinematic chain is represented by graph in which links in the chain become vertices and joints become edges. The degree of a vertex of an ideal graph with ' $n$ ' vertices will be ' $n-1$ '. The connectivity of every vertex is represented by a fuzzy number assigned to it in the range of 0 to 1 , which are called 'crisp numbers'. The number ' 0 ' indicates that the vertex is isolated or not connected to any other vertex. The number ' 1 ' indicates that the vertex is fully connected to all other vertices. In practice, both the extreme values are not attained. Actual values will have intermediate values between 0 and 1 .

For a graph with ' $n$ ' vertices, the connectivity index of a vertex ' $i$ ' will be $n_{i} /(n-1)$

Where $n_{i}$ : Number of vertices connected to link ' $i$ ' and $n$ : Total number of vertices or links

The connectivity indices of all the links form a set of fuzzy numbers also called as 'fits'. Matrices will be formed for each kinematic chain based on these fits. Each such matrix indicates the extent of adjacency a link has with other links.

To explain the procedure, consider an 8-link 1-dof kinematic chain shown in Figures 1(a) and its graph in Figure $1(b)$.

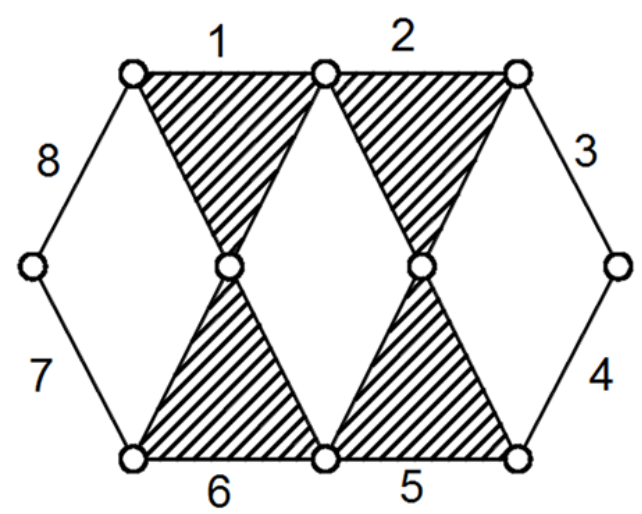

FIGURE 1(a): KINEMATIC CHAIN NO.1 IN APPENDIX I

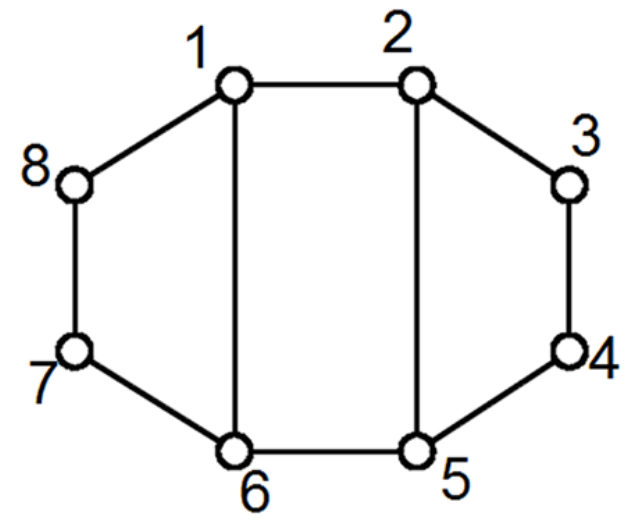

FIGURE 1(b): GRAPH FOR THE KINEMATIC CHAIN IN FIGURE 1(a) 
The fits for vertices $1,2,3,4,5,6,7$ and 8 are given by $3 / 7,3 / 7,2 / 7,2 / 7,3 / 7,3 / 7,2 / 7,2 / 7$ or $0.42,0.42,0.28,0.28,0.42,0.42,0.28,0.28$.

\section{RATING OF KinEMATIC ChAINS USING FUZZY ENTROPY}

Structural comparison and rating of kinematic chains is to be done stage by stage. In Stage I, basic fuzzy entropy is calculated for each kinematic chain. As we note from basic definition of fuzzy entropy, the chain with less entropy is more preferred as it will have less uncertainty.

\section{(a) STAGE I:}

After assigning the fuzzy parameters to each link, basic fuzzy entropy is calculated for each kinematic chain. Sorting of kinematic chains in the ascending order of entropy is done.

It is noted from the output of Stage I that the kinematic chains with same linkage characteristics (number of binary links, ternary links etc.) will have same fuzzy entropy which need to be sorted in next stage. It is observed that kinematic chains 1-9 in Appendix I have same basic entropy.

\section{(b) STAGE II:}

In this stage, adjacency matrices are prepared at different levels. For any two distinct kinematic chains, the adjacency of links will certainly differ. Basing on the level of adjacency with all other links, adjacency matrices at different levels are prepared. The input for the entropy calculation is from adjacency matrices only.

\section{Preparation Of Adjacency Matrix}

Adjacency matrices are generated in various levels i.e., first level, second level, third level etc. First level means adjacency of links that are directly connected to another link or in other words separated by one edge or joint only. Second level means adjacency of links that are separated by two joints or edges. The same applies to third and fourth level. For each level, adjacency matrices $\mathrm{A}_{1}, \mathrm{~A}_{2}, \mathrm{~A}_{3}$ etc. are prepared.

\section{FIRST LEVEL ADJACENCY MATRIX $\left(A_{1}\right)$}

First level adjacency matrix consists of fits or fuzzy elements corresponding to their direct connectivity. For example, for link 1 , fuzzy vector (collection of fuzzy values for a link) will be $0,0.42,0,0,0,0.42,0,0.28$ as link 1 is connected to links 2,6 and 8 only. Fuzzy elements of connected links will be copied from (1). Fits of other links will be 0 . Similarly, fuzzy vectors of all links are prepared to generate First level adjacency matrix is given by $\mathrm{A}_{1}$.

$$
\mathrm{A}_{1}=\left(\begin{array}{cccccccc}
0 & 0.42 & 0 & 0 & 0 & 0.42 & 0 & 0.28 \\
0.42 & 0 & 0.28 & 0 & 0.42 & 0 & 0 & 0 \\
0 & 0.42 & 0 & 0.28 & 0 & 0 & 0 & 0 \\
0 & 0 & 0.28 & 0 & 0.42 & 0 & 0 & 0 \\
0 & 0.42 & 0 & 0.28 & 0 & 0.42 & 0 & 0 \\
0.42 & 0 & 0 & 0 & 0.42 & 0 & 0.28 & 0 \\
0 & 0 & 0 & 0 & 0 & 0.42 & 0 & 0.28 \\
0.42 & 0 & 0 & 0 & 0 & 0 & 0.28 & 0
\end{array}\right)
$$

\section{SECOND LEVEL ADJACENCY MATRIX $\left(A_{2}\right)$}

Second level adjacency matrix consists of fits or fuzzy elements corresponding to their second level connectivity i.e. links that are separated by two edges or two joints in the graph.

For example, the first row corresponds to fuzzy vector of link at second level, which consists of fits for links 3 , 5 and 7 only as these links are separated from link 1 by two joints. All the other fits will be zero. For the kinematic chain shown in FIGURE 1(a), second level adjacency matrix is given by $\mathrm{A}_{2}$. 


$$
\mathrm{A}_{2}=\left(\begin{array}{cccccccc}
0 & 0 & 0.28 & 0 & 0.42 & 0 & 0.28 & 0 \\
0 & 0 & 0 & 0.28 & 0 & 0.42 & 0 & 0.28 \\
0.42 & 0 & 0 & 0 & 0.42 & 0 & 0 & 0 \\
0 & 0.42 & 0 & 0 & 0 & 0.42 & 0 & 0 \\
0.42 & 0 & 0.28 & 0 & 0 & 0 & 0.28 & 0 \\
0 & 0.42 & 0 & 0.28 & 0 & 0 & 0 & 0.28 \\
0.42 & 0 & 0 & 0 & 0.42 & 0 & 0 & 0 \\
0 & 0.42 & 0 & 0 & 0 & 0.42 & 0 & 0
\end{array}\right)
$$

\section{THIRD LEVEL ADJACENCY MATRIX $\left(A_{3}\right)$}

Third level adjacency matrix consists of fits or fuzzy elements corresponding to their third level connectivity i.e. links that are separated by three edges or three joints in the graph. For example, the first row corresponds to fuzzy vector of link at third level consists of fits for link 4 only as link 4 only is separated from link 1 by three joints. All the other fits will be zero. For the kinematic chain in FIGURE 1(a), third level adjacency matrix is given by $\mathrm{A}_{2}$.

$$
\mathrm{A}_{3}=\left(\begin{array}{cccccccc}
0 & 0 & 0 & 0.28 & 0 & 0 & 0 & 0 \\
0 & 0 & 0 & 0 & 0 & 0 & 0.28 & 0 \\
0 & 0 & 0 & 0 & 0 & 0.42 & 0 & 0.28 \\
0.42 & 0 & 0 & 0 & 0 & 0 & 0.28 & 0 \\
0 & 0 & 0 & 0 & 0 & 0 & 0 & 0.28 \\
0 & 0 & 0.28 & 0 & 0 & 0 & 0 & 0 \\
0 & 0.42 & 0 & 0.28 & 0 & 0 & 0 & 0 \\
0 & 0 & 0.28 & 0 & 0.42 & 0 & 0 & 0
\end{array}\right)
$$

\section{FOURTH OR HIGHER LEVEL ADJACENCY MATRICES}

In higher linkage kinematic chains, adjacency of links separated by three joints, four joints etc. by least path in the graph are taken and corresponding level adjacency matrices will be formed in the same procedure as explained earlier for first, second and third levels.

\section{ALGORITHM TO CALCULATE ENTROPY}

1. Initially, First level adjacency matric is considered. Fuzzy vector for each link will be the corresponding row elements in the first level adjacency matrix.

2. Basic fuzzy entropy is calculated for each link in the similar lines of entropy calculation in Stage I.

3. Summation of basic entropy values of all the links will result Fuzzy entropy of total chain at the first level adjacency which can be designed as $\mathrm{F}_{1}$

4. Sorting of kinematic chains for the groups which have similar values in Stage I is carried out by comparison of $F_{1}$ values.

5. If any two kinematic chains results same $F_{1}$ value calculate fuzzy entropy value considering Second level adjacency values.

6. The algorithm is explained by taking one example. 


\section{Example 1:}

Consider a kinematic chain shown in Figure 2 (Chain No. 2 in the Appendix I )

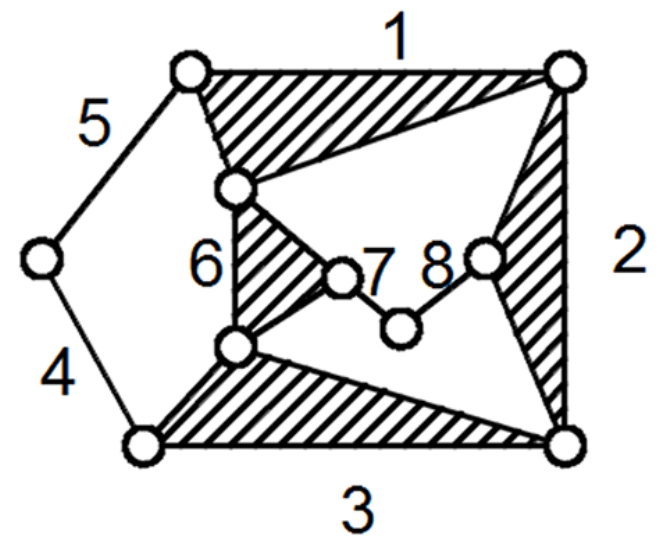

FiguRE 2: KinEMATIC CHAIN No. 2 IN APPENDIX I

The fuzzy parameters for the chain will be: [ $3 / 7,2 / 7,2 / 7,3 / 7,3 / 7,2 / 7,2 / 7,3 / 7]$ or $[0.42,0.28,0.28,0.42,0.42,0.28,0.28,0.42]$

The first level adjacency matrix is given by $\mathrm{A}_{1}$.

$$
\mathrm{A}_{1}=\left(\begin{array}{cccccccc}
0 & 0.28 & 0 & 0 & 0.42 & 0 & 0 & 0.42 \\
0.42 & 0 & 0.28 & 0 & 0 & 0 & 0 & 0 \\
0 & 0.28 & & 0.42 & 0 & 0 & 0 & 0 \\
0 & 0 & 0.28 & 0 & 0.42 & 0 & 0 & 0.42 \\
0.42 & 0 & 0 & 0.42 & 0 & 0.28 & 0 & 0 \\
0 & 0 & 0 & 0 & 0.42 & & 0.28 & 0 \\
0 & 0 & 0 & 0 & 0 & 0.28 & 0 & 0.42 \\
0.42 & 0 & 0 & 0.42 & 0 & 0 & 0.28 & 0
\end{array}\right)
$$

From the matrix $A_{1}$ fuzzy vector for the Link 1 will be elements of first row i.e.

$\left[\begin{array}{llllllll}0 & 0.28 & 0 & 0 & 0.42 & 0 & 0 & 0.42\end{array}\right]$

The Nearest crisp vector will be

$\left[\begin{array}{llllllll}0 & 0 & 0 & 0 & 0 & 0 & 0 & 0] \text { and }\end{array}\right.$

Farthest crisp vector will be

$\left[\begin{array}{llllllll}1 & 1 & 1 & 1 & 1 & 1 & 1 & 1\end{array}\right]$

Nearest distance vector will be

$\left[\begin{array}{llllllll}0 & 0.28 & 0 & 0 & 0.42 & 0 & 0 & 0.42\end{array}\right]$ and

Farthest distance vector will be
$\left[\begin{array}{llllllll}1 & 0.72 & 1 & 1 & 0.58 & 1 & 1 & 0.58\end{array}\right]$

The nearest distance $=0+0.28+0+0+0.42+0+0+0.42=1.12$

The farthest distance $=1+0.72+1+1+0.58+1+1+0.58=6.88$

The fuzzy entropy of Link 1 at first adjacency level $=1.12 / 6.88=0.1628$

Similarly, fuzzy entropy for all other links is calculated. The results are presented in TABLE 1. 
TABLE 1: ENTROPY CALCULATIONS OF A KINEMATIC CHAIN

\begin{tabular}{|c|c|c|c|c|c|c|c|c|c|c|}
\hline \multirow{9}{*}{$\begin{array}{l}\text { Nearest distance } \\
\text { matrix }\end{array}$} & Link & 1 & 2 & & 3 & 4 & 5 & 6 & 7 & 8 \\
\hline & 1 & 0 & 0 & & 0 & 0 & 0 & 0 & 0 & 0 \\
\hline & 2 & 0 & 0 & & 0 & 0 & 0 & 0 & 0 & 0 \\
\hline & 3 & 0 & 0 & & 0 & 0 & 0 & 0 & 0 & 0 \\
\hline & 4 & 0 & 0 & & 0 & 0 & 0 & 0 & 0 & 0 \\
\hline & 5 & 0 & 0 & & 0 & 0 & 0 & 0 & 0 & 0 \\
\hline & 6 & 0 & 0 & & 0 & 0 & 0 & 0 & 0 & 0 \\
\hline & 7 & 0 & 0 & & 0 & 0 & 0 & 0 & 0 & 0 \\
\hline & 8 & 0 & 0 & & 0 & 0 & 0 & 0 & 0 & 0 \\
\hline \multirow{9}{*}{$\begin{array}{l}\text { Nearest distance } \\
\text { difference matrix }\end{array}$} & Link & 1 & 2 & 3 & 4 & 5 & 6 & 7 & 8 & Total \\
\hline & 1 & 0 & 0.28 & 0 & 0 & 0.42 & 0 & 0 & 0.42 & 1.12 \\
\hline & 2 & 0.42 & 0 & 0.28 & 0 & 0 & 0 & 0 & 0 & 0.7 \\
\hline & 3 & 0 & 0.28 & 0 & 0.42 & 0 & 0 & 0 & 0 & 0.7 \\
\hline & 4 & 0 & 0 & 0.28 & 0 & 0.42 & 0 & 0 & 0.42 & 1.12 \\
\hline & 5 & 0.42 & 0 & 0 & 0.42 & 0 & 0.28 & 0 & 0 & 1.12 \\
\hline & 6 & 0 & 0 & 0 & 0 & 0.42 & 0 & 0.28 & 0 & 0.7 \\
\hline & 7 & 0 & 0 & 0 & 0 & 0 & 0.28 & 0 & 0.42 & 0.7 \\
\hline & 8 & 0.42 & 0 & 0 & 0.42 & 0 & 0 & 0.28 & 0 & 1.12 \\
\hline \multirow{9}{*}{$\begin{array}{c}\text { Farthest distance } \\
\text { matrix }\end{array}$} & Link & 1 & 2 & & 3 & 4 & 5 & 6 & 7 & 8 \\
\hline & 1 & 1 & 1 & & 1 & 1 & 1 & 1 & 1 & 1 \\
\hline & 2 & 1 & 1 & & 1 & 1 & 1 & 1 & 1 & 1 \\
\hline & 3 & 1 & 1 & & 1 & 1 & 1 & 1 & 1 & 1 \\
\hline & 4 & 1 & 1 & & 1 & 1 & 1 & 1 & 1 & 1 \\
\hline & 5 & 1 & 1 & & 1 & 1 & 1 & 1 & 1 & 1 \\
\hline & 6 & 1 & 1 & & 1 & 1 & 1 & 1 & 1 & 1 \\
\hline & 7 & 1 & 1 & & 1 & 1 & 1 & 1 & 1 & 1 \\
\hline & 8 & 1 & 1 & & 1 & 1 & 1 & 1 & 1 & 1 \\
\hline
\end{tabular}




\begin{tabular}{|c|c|c|c|c|c|c|c|c|c|c|}
\hline \multirow{9}{*}{$\begin{array}{l}\text { Farthest distance } \\
\text { difference matrix }\end{array}$} & Link & 1 & 2 & 3 & 4 & 5 & 6 & 7 & 8 & Total \\
\hline & 1 & 1 & 0.72 & 1 & 1 & 0.58 & 1 & 1 & 0.58 & 6.88 \\
\hline & 2 & 0.58 & 1 & 0.72 & 1 & 1 & 1 & 1 & 1 & 7.3 \\
\hline & 3 & 1 & 0.72 & 1 & 0.58 & 1 & 1 & 1 & 1 & 7.3 \\
\hline & 4 & 1 & 1 & 0.72 & 1 & 0.58 & 1 & 1 & 0.58 & 6.88 \\
\hline & 5 & 0.58 & 1 & 1 & 0.58 & 1 & 0.72 & 1 & 1 & 6.88 \\
\hline & 6 & 1 & 1 & 1 & 1 & 0.58 & 1 & 0.72 & 1 & 7.3 \\
\hline & 7 & 1 & 1 & 1 & 1 & 1 & 0.72 & 1 & 0.58 & 7.3 \\
\hline & 8 & 0.58 & 1 & 1 & 0.58 & 1 & 1 & 0.72 & 1 & 6.88 \\
\hline \multirow{9}{*}{$\begin{array}{l}\text { Fuzzy entropy of } \\
\text { links of kinematic } \\
\text { chain }\end{array}$} & & & & \multicolumn{2}{|c|}{ Link } & \multicolumn{2}{|c|}{ Entropy } & & & \\
\hline & & & & \multicolumn{2}{|l|}{1} & \multicolumn{2}{|c|}{0.1628} & & & \\
\hline & & & & \multicolumn{2}{|l|}{2} & \multicolumn{2}{|c|}{0.0959} & & & \\
\hline & & & & \multicolumn{2}{|l|}{3} & \multicolumn{2}{|c|}{0.0959} & & & \\
\hline & & & & \multicolumn{2}{|l|}{4} & \multicolumn{2}{|c|}{0.1628} & & & \\
\hline & & & & \multicolumn{2}{|l|}{5} & \multicolumn{2}{|c|}{0.1628} & & & \\
\hline & & & & \multicolumn{2}{|l|}{6} & \multicolumn{2}{|c|}{0.0959} & & & \\
\hline & & & & \multicolumn{2}{|l|}{7} & \multicolumn{2}{|c|}{0.0959} & & & \\
\hline & & & & \multicolumn{2}{|l|}{8} & \multicolumn{2}{|c|}{0.1628} & & & \\
\hline
\end{tabular}

\section{RESULTS}

All the 8-link 1-dof kinematic chains (16 no.) shown in Appendix I are sorted and ranking is done by considering basic entropy, first level adjacency and second level adjacency. All the values are presented in Appendix II. It is observed from the results that the sorting of 8-link 1-dof kinematic chains necessitates adjacency matrices up-to second level only.

\section{CONCLUSION}

Structural comparison of 8-link 1-DOF kinematic chains (16 no.) is done by using the proposed method efficiently with fewer computations. The proposed method is very easy to understand and implement. It can be automated by using a computer program by giving the adjacency values as input to get the rating of kinematic chains as output. The proposed method can be applied to higher linkages and more degree of freedom i.e. 9-link 2-dof, 10-link 1-dof etc.

\section{REFERENCES}

[1] A. C. Rao and C. N. Rao, "Loop based pseudo hamming values-I testing isomorphism and rating kinematic chains", Mechanism and Machine Theory, Vol. 28, pp: 113-127, 1993.

[2] A. C. Rao and C. N. Rao, "Loop based pseudo hamming values-II inversions, preferred frames and actuators", Mechanism and Machine Theory, Vol. 28, pp: 129-143, 1993.

[3] A. C. Rao and C. N. Rao, "Selection of best frame, input and output links for function generators modelled as probabilistic systems", Mechanism and Machine Theory, Vol. 31(7), pp: 113-127, 1996.

[4] A. C. Rao, "Topology based rating of kinematic chains and inversions using information theory", Mechanism and Machine Theory, Vol.33, pp: 1055-1062, 1998.

[5] A. B. S. Rao, A. C. Rao and A. Srinath, "Synthesis of Planar Kinematic Chains", Institution of Engineers Journal, Vol. 86, pp: 195201, 2006.

[6] G.S. Bedi, S. Sanyal, "Modified joint connectivity approach for identification of topological characteristics of planar kinematic chains", Proceedings of the Institution of Mechanical Engineers, Part C: Journal of Mechanical Engineering Science, Vol. 225, pp: 2700-2717, 2011.

[7] G.S. Bedi,S. Sanyal, "Structure based grading of Kinematic Chains", Applied Mechanics and Materials, Vol. 575, pp: 501-506, 2014. 
[8] Huafeng Ding, Fengmao Hou, Andres Kecskemethy and Zhen Huang, "Synthesis of the whole family of planar 1-DOF kinematic chains and creation of their atlas databases", Mechanism and Machine Theory, Vol. 47 (1), pp: 1-15, 2012.

[9] Huafeng Ding, Peng Huang, Jinfang Liu and Andres Kecskemethy, "Automatic structural synthesis of the whole family of planar 3DOF closed loop mechanisms", ASME Journal of Mechanisms and Robotics, Vol. 5(4), pp:1-10, 2013.

[10] Huafeng Ding, Bin Zi, Peng Huang,Andrés Kecskeméthy, "The Whole Family of kinematic structures for planar 2-and 3-DOF fractionated kinematic chains", Mechanism and Machine Theory, Vol.70, pp: 74-90, 2013.

[11] Chang Z. Zhang C., Yang Y., Wang Y, “A New Method to Mechanism Kinematic Chain Isomorphism Identification”, Mechanism and Machine Theory, Vol. 37, pp: 411-417, 2002.

[12] Hong-Sen Yan, Yu-Ting Chiu, "On the number synthesis of kinematic chains", Mechanism and Machine Theory, Vol.89, pp: 128$144,2015$.

[13] T.S. Mruthyunjaya and M.R. Raghavan, "Structural Analysis of Kinematic Chains and Mechanisms Based on Matrix Representation”, ASME Journal of Mech. Design, Vol. 101(3), pp: 488-494, 1979.

[14] T.S. Mruthyunjaya, "A computerized methodology for structural synthesis of kinematic chains - Part I, II and III", Mechanism and Machine Theory, Vol. 14, pp: 487-530, 1984.

[15] T.S. Mruthyunjaya and H. R. Balasubramanian, "In quest for reliable and efficient test for detection of isomorphism in kinematic chains", Mechanism and Machine Theory, Vol. 10(2), pp: 131-139, 1987.

[16] H. Ding and H. Zhen, "A new theory for the topological structure analysis of kinematic chains and its Applications", Mechanism and Machine Theory, Vol. 42(10), pp: 1264-1279, 2007.

[17] Robert Simoni and Daniel Martins, "Criteria for Structural Synthesis and Classification of Mechanisms", Proceedings of COBEM 2007, $19^{\text {th }}$ International Congress of Mech. Engg., Brasilia, DF, Nov.5-9, 2007.

[18] Mechael Chester, "Neural Networks, A tutorial”, PTR Prentice Hall, New-Jersey, pp: 119- 130, 1993. 


\section{APPENDIX I}

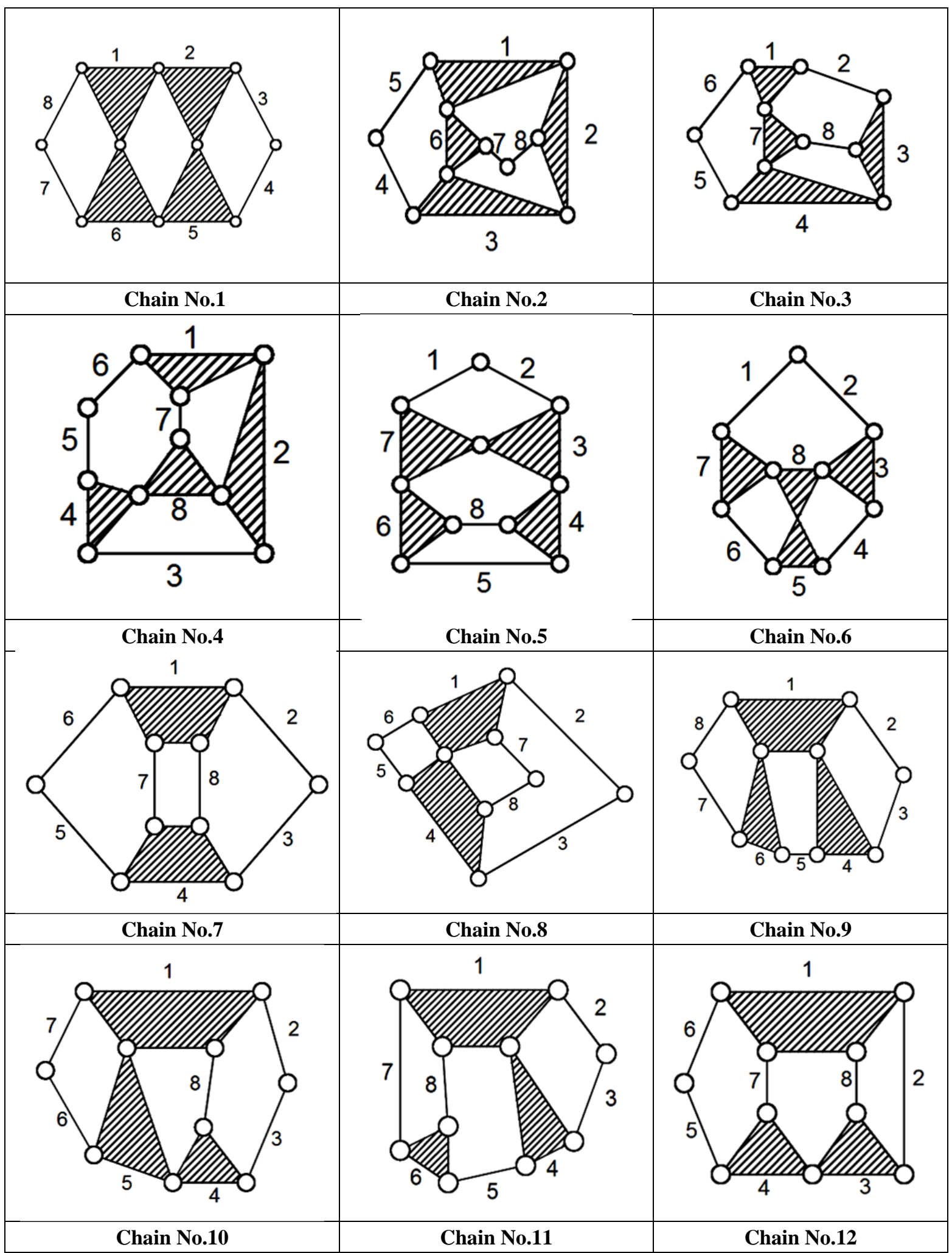




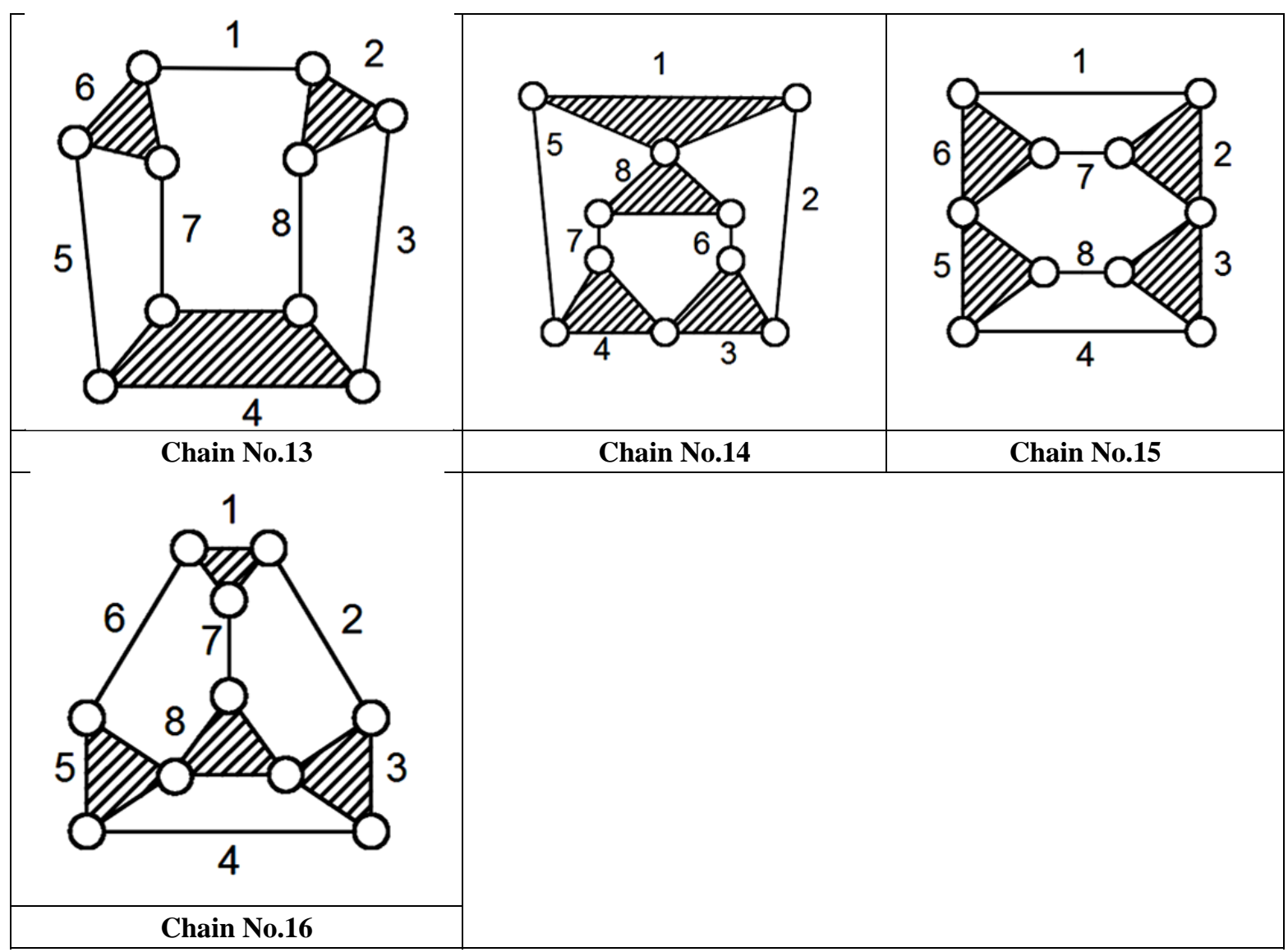




\section{APPENDIX II}

\begin{tabular}{|c|c|c|c|c|}
\hline $\begin{array}{c}\text { CHAIN } \\
\text { NUMBER }\end{array}$ & $\begin{array}{c}\text { BASIC } \\
\text { ENTROPY } \\
\text { 'F' }\end{array}$ & $\begin{array}{c}\text { ENTROPY } \\
\text { @FIRST } \\
\text { ADJACENCY } \\
\text { 'F1' }\end{array}$ & $\begin{array}{c}\text { ENTROPY } \\
\text { @SECOND } \\
\text { ADJACENCY } \\
\text { 'F2' }\end{array}$ & RANK \\
\hline 1 & 0.714 & 1.0347 & 1.0277 & 8 \\
\hline 2 & 0.714 & 1.0347 & 1.4955 & 9 \\
\hline 3 & 0.714 & 1.0312 & 1.4734 & 6 \\
\hline 4 & 0.714 & 1.0312 & 1.2096 & 5 \\
\hline 5 & 0.714 & 1.0312 & 1.1901 & 4 \\
\hline 6 & 0.714 & 1.0322 & 1.2801 & 7 \\
\hline 7 & 0.714 & 1.0286 & 1.4251 & 3 \\
\hline 8 & 0.714 & 0.9633 & 1.2096 & 1 \\
\hline 9 & 0.714 & 1.0277 & 1.648 & 2 \\
\hline 10 & 0.745 & 0.9903 & 1.1496 & 10 \\
\hline 11 & 0.745 & 1.0357 & 1.4924 & 14 \\
\hline 12 & 0.745 & 0.9939 & 1.1754 & 11 \\
\hline 13 & 0.745 & 0.9965 & 1.2656 & 12 \\
\hline 14 & 0.745 & 0.9985 & 1.0143 & 13 \\
\hline 15 & 0.778 & 0.9618 & 1.1534 & 16 \\
\hline 16 & 0.778 & 0.9561 & 1.4615 & 15 \\
\hline
\end{tabular}




\section{AUTHORS’ PROFILE}

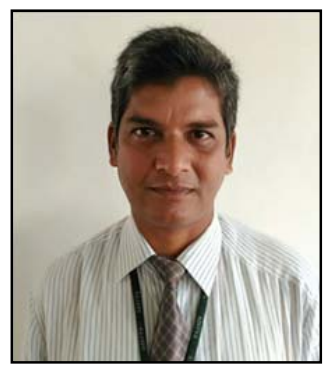

Vallem Srinivasa Rao is presently working as an Associate Professor in the Department of Mechanical Engineering, Aditya Engineering College, Surampalem, Andhra Pradesh, India. He is currently pursuing his Ph.D from Jawaharlal Nehru Technological University (JNTUK), Kakinada, Andhra Pradesh, India. He completed his Bachelor degree in Mechanical Engineering from JNTU, Hyderabad. Master Degree in Machine Design from JNTU, Hyderabad. He has 15 years of teaching experience. His areas of interest are Synthesis and Analysis of Kinematic Chains.

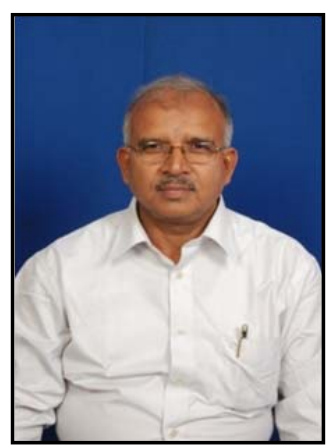

Kuchibhotla Mallikarjuna Rao is presently working as Professor in the Department of Mechanical Engineering, University College of Engineering, Jawaharlal Nehru Technological University, Kakinada (JNTUK), Andhra Pradesh, India. He has completed his Bachelor Degree in National Institute of Technology(NIT), Warangal, Telangana, India, Master Degrees in Asian Institute of Technology(AIT), Bangkok, Thailand and Indian Institute of Sciences (IISc), Bangalore, India and Doctoral Degree in Indian Institute of Sciences (IISc), Bangalore, India. He has 30 years of Teaching and research experience. He presented more than 70 papers in various Journals and Conferences. He has guided more than 60 P.G Scholars and also 22 Ph.D. Scholars. He is a Fellow of Institution of Engineers, India, Chairman and Member of Board of Studies, Mechanical Engineering, JNTUK, Kakinada and also a Life Member of Indian Society for Technical Education, India. His research area is Finite Element Method.

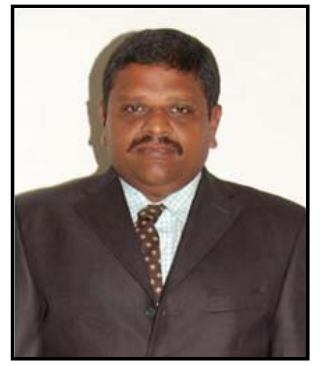

Annambhotla Balaji Srinivasa Rao is presently working as Principal Sri Vasavi Institute of Engineering and Technology, Nandamuru, Andhra Pradesh, India. He obtained his Bachelor degree in Mechanical Engineering from Nagarjuna University, Guntur, Andhra Pradesh, India, Master Degree in Production Engineering from University College of Engineering, Osmania University, Hyderabad, India and Doctoral Degree from Osmania University, Hyderabad, India. He has 20 years of teaching and research experience and organized several National \& International Symposiums. He has published more than 25 papers in various Journals and Conferences. He is a life member of Indian Society for Technical Education, India. His area of Research is Robotics. 